\title{
Step-Down From Multiple- to Single-Dose Proton Pump Inhibitors (PPIs): A Prospective Study of Patients With Heartburn or Acid Regurgitation Completely Relieved With PPIs
}

\author{
John M. Inadomi, M.D., Lisa McIntyre, Pharm.D., Latoya Bernard, M.P.H., and A. Mark Fendrick, M.D. \\ Department of Veterans Affairs Center for Practice Management and Outcomes Research, Ann Arbor; \\ Division of Gastroenterology, Department of Medicine, and the Consortium for Health Outcomes, Innovation, \\ and Cost-Effectiveness Studies, University of Michigan School of Medicine, Ann Arbor, Michigan
}

OBJECTIVES: Management costs for gastroesophageal reflux disease are high because of the expensive medications used for maintenance therapy. Previous studies have illustrated the success of step-down from proton pump inhibitors (PPIs) to less-expensive therapy once symptoms have abated. This study was conducted to determine whether patients requiring greater than single-dose PPI for initial symptom resolution could be stepped-down to single-dose PPI and whether this intervention decreased costs or adversely affected quality of life.

METHODS: Consecutive patients in whom greater than single-dose PPI had completely alleviated reflux-type symptoms (heartburn or acid regurgitation) were recruited through the use of pharmacy records of PPI prescriptions. Eligible subjects completed baseline demographic information and quality-of-life surveys and were stepped-down to single-dose PPI (lansoprazole $30 \mathrm{mg}$ or omeprazole $20 \mathrm{mg}$ daily). Follow-up continued for 6 months or until subjects reported recurrence of reflux-type symptoms, at which point PPIs were reinstituted at the dose that had originally alleviated the subjects' symptoms. The primary outcome was the proportion of subjects in whom step-down was successful, defined as no recurrence of reflux-type symptoms on single-dose PPI.

RESULTS: A total of 117 subjects enrolled in the study; all were followed to the primary endpoint. $79.5 \%$ did not report recurrent symptoms of heartburn or acid regurgitation during the 6 months after step-down to single-dose PPI. Logistic regression revealed that longer duration of PPI use before study enrollment was associated with greater likelihood of symptom recurrence with step-down. Although quality of life was not significantly altered, dyspepsia (excluding reflux-type symptoms) increased. Overall costs of management were reduced.

CONCLUSIONS: The majority of patients rendered asymptomatic on greater than single-dose PPI might be subsequently stepped-down to single-dose therapy without recur- rence of reflux-type symptoms. This intervention can decrease management costs without adversely affecting quality of life. (Am J Gastroenterol 2003;98:1940-1944. () 2003 by Am. Coll. of Gastroenterology)

\section{INTRODUCTION}

Considerable resources are required to manage gastroesophageal reflux disease (GERD). Proton pump inhibitors (PPIs) are the greatest outpatient pharmacy expenditure in the U.S. Department of Veterans Affairs (VA) nationwide, accounting for greater than $\$ 100$ million annually (1), this despite deep discounts in the procurements costs of formulary PPIs. Additionally, the proportion of patients prescribed greater than single-dose PPI is as high as $30 \%$. It is not known whether the use of greater than single-dose PPI is due to the presence of severe disease, provider prescribing habits, or inability of the formulary drugs to control symptoms with lower doses.

Long-term PPI therapy might not be required for all GERD patients. Histamine ${ }_{2}$-receptor antagonists (H2RAs) have also been shown to effectively treat GERD symptoms and heal esophagitis (2-4). Additionally, we have previously shown that in certain settings, the majority of patients prescribed PPIs for GERD symptoms (heartburn or acid regurgitation) remain asymptomatic despite discontinuation of PPIs and institution of less-expensive medication, such as H2RAs or no medication (5). Finally, not all patients prescribed PPIs for GI complaints have GERD; a sizable number have functional dyspepsia that might respond to different strategies of acid suppression (6-10).

We wished to examine the group of patients at our medical center who had reflux-type symptoms completely relieved with greater than single-dose PPI. The objective of this study was to determine the feasibility of stepping-down patients from greater than single-dose PPI to single-dose therapy and to assess the impact of this intervention on management costs and patient quality of life. 


\section{MATERIALS AND METHODS}

The population studied was composed of patients receiving greater than single-dose PPI, defined as greater than lansoprazole $30 \mathrm{mg}$ daily or omeprazole $20 \mathrm{mg}$ daily, for the treatment of heartburn or acid regurgitation from the VA Ann Arbor Healthcare System. This system encompasses several outpatient facilities in Michigan and Ohio, as well as the main medical center in Ann Arbor, Michigan. Patients were identified from a list generated through the VA computerized pharmacy database (pharmacy module of VISTA). Because PPIs might be prescribed for short periods of treatment for non-GERD indications, such as Helicobacter pylori eradication, only prescriptions filled for greater than 8 wk were considered. The process of PPI step-down was a mandated part of clinical care; however, completion of research materials, including quality-of-life instruments, required informed consent. The study was approved by the local research and human studies committees.

Identified patients were invited to attend a medical specialty clinic, staffed by a clinical pharmacist under the supervision of a gastroenterologist (the Heartburn Clinic). During the initial visit, patients were interviewed, and their PPI regimen and indication for treatment was assessed. Patients were eligible for the study if they were taking greater than single-dose PPI and had denied symptoms of heartburn or acid regurgitation on a stable dose of medication for at least $8 \mathrm{wk}$. Dyspepsia could be present and could have been the dominant or most bothersome symptom; however, patients complaining of dyspepsia in the absence of heartburn or acid regurgitation were not enrolled. Other exclusion criteria included 1) weight loss, 2) evidence of GI bleeding, 3) dysphagia, 4) extraesophageal disease related to acid regurgitation, such as pulmonary or laryngeal disease, and 5) non-GERD esophageal or gastric pathology, such as peptic ulcer, varices, esophageal malignancy, acid hypersecretory states, or motility disorders.

After enrolling in the study, subjects were asked to complete two quality-of-life instruments; the 12-Item Short Form Health Survey (SF-12), to assess generic quality of life, and a validated disease-specific measure (11). Additional information, including demographic data, height and weight measurements to assess body mass index (BMI), comorbid conditions, concurrent medication use, initial GERD symptoms before therapy, and previous GERD medication were collected at the initial visit.

PPI step-down was performed according to the following strategy. Eligible subjects were prescribed single-dose PPI (lansoprazole $30 \mathrm{mg}$ or omeprazole $20 \mathrm{mg}$ p.o. once daily $1 / 2$ $\mathrm{h}$ before breakfast) at the initial visit and returned for follow-up 2 wk after initiation of step-down therapy. Subjects in whom heartburn or acid regurgitation had not recurred continued on single-dose PPI and were followed at 3-month intervals in the Heartburn Clinic to assess symptom recurrence and were also instructed to contact the clinical pharmacist in the interim if heartburn or acid regurgitation recurred. Subjects in whom symptoms of heartburn or acid regurgitation recurred were placed back on their original PPI dose.

Six months subsequent to the initial visit, the diseasespecific and generic quality-of-life instruments were readministered. The primary outcome variable was the proportion of subjects who were successfully stepped-down to single-dose PPI therapy, defined as having no recurrence of heartburn or acid regurgitation 6 months after PPI stepdown. Secondary outcomes included changes in healthrelated quality of life or pharmacy expenditures, and predictors of successful step-down.

\section{Data Analysis}

Descriptive statistics were used to characterize the study population and measure the primary outcome (proportion of subjects successfully stepped-down to single-dose PPI).

To determine the predictors of successful step-down, univariate and multivariable logistic regression analyses were planned. Variables found in the univariate analysis to be significantly associated with successful step-down ( $p$ value $<0.05$ ), were included in a multivariable logistic regression analysis. Changes in quality of life and disease severity were analyzed with paired $t$ tests to assess the effect of the intervention on these variables. Based on previous data concerning PPI step-down management in GERD patients (5), 100 subjects would allow inclusion of five predictor variables into the final logistic model (12-14).

Cost analysis was limited to the cost of medications assessed from pharmacy expenditures by the medical center and direct operating cost of the Heartburn Clinic, including the salary and benefits of the clinical pharmacist staffing the clinic.

\section{RESULTS}

A total of 298 consecutive patients were evaluated in the Heartburn Clinic. Of these, 126 (42.3\%) were eligible for step-down. The other 172 were ineligible for the following reasons: already taking single-dose PPI (81 patients), persistent heartburn or acid regurgitation on current PPI dose (54), not taking PPI for GERD (19), complicated reflux disease, including strictures (8), alarm symptoms requiring additional evaluation (7), esophageal cancer (2), and gastric cancer (1). Nine (7.1\%) of the eligible patients refused to participate in the study, resulting in a total of 117 subjects who enrolled in the study. Follow-up to the primary outcome was achieved in all these 117 subjects.

Table 1 presents the patients' baseline characteristics on the variables of age, gender, cigarette use, alcohol use, BMI, initial PPI, and visit site. The mean age was $64.8 \mathrm{yr}$, and the majority were male, nonsmokers, and did not drink alcohol. Eighty-four patients had undergone esophagogastroduodenoscopy, and four had 24-h ambulatory $\mathrm{pH}$ monitoring studies before study enrollment. 
Table 1. Study Population Characteristics

\begin{tabular}{lc}
\hline \multicolumn{1}{c}{ Characteristic } & Value $(\%)$ \\
\hline Age (yr) & \\
Mean & 64.8 \\
Median & 66 \\
Range & $36-84$ \\
Gender & \\
Female & $5(4.3)$ \\
Male & $112(95.7)$ \\
Tobacco use & \\
Current smokers & $31(26.5)$ \\
Current nonsmokers & $86(73.5)$ \\
Alcohol use & $35(29.9)$ \\
Current drinkers & $82(70.1)$ \\
Current nondrinkers & $18(16.4)$ \\
BMI & $54(49.1)$ \\
Normal or below normal (15-24.9) & $38(34.5)$ \\
Overweight (25-29.9) & \\
Obese (>30) & $103(88.0)$ \\
Initial PPI & $14(12.0)$ \\
Lansoprazole & \\
Omeprazole & $76(65.0)$ \\
Visit site & $41(35.0)$ \\
Ann Arbor & \\
Toledo/other & \\
\hline
\end{tabular}

Of the 117 patients participating in the study, 93 (79.5\%) remained successfully stepped-down after the 6-month follow-up period (Fig. 1). Of those in whom step-down was unsuccessful, the mean failure time (time to reinstitution of original dose of PPIs) was 49.6 days (median 28 days, range 2-280 days). Of the 24 failures, 23 occurred within the first 3 months of follow-up.

Subgroup analysis was performed to determine whether the prior pattern of PPI use impacted the ability to stepdown to single-dose therapy (Table 2). Of the subjects in the study who had originally been prescribed single-dose PPI before escalation of dose, $77.1 \%$ successfully stepped-down to single-dose PPI without recurrence of heartburn or acid regurgitation. Of the subjects who had never been on singledose therapy, $82.1 \%$ were successfully stepped-down. There were 19 subjects who had previously attempted step-down

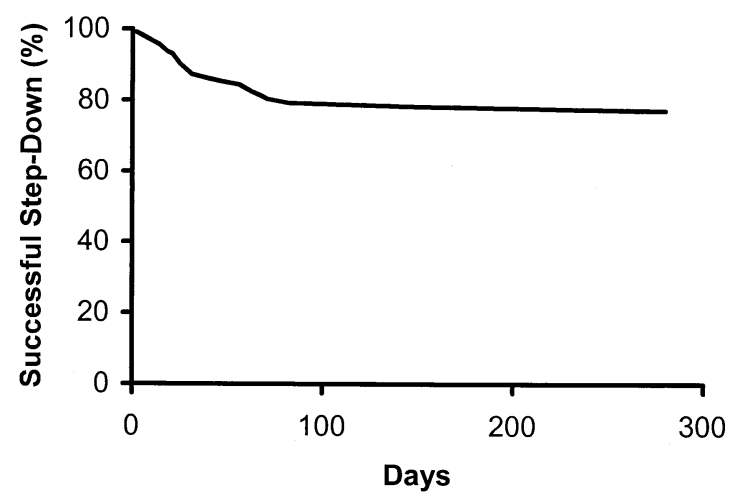

Figure 1. Proportion of subjects without recurrent GERD symptoms after PPI step-down.
Table 2. Subgroup Analysis: Previous PPI Use

\begin{tabular}{lcc}
\hline \multicolumn{1}{c}{ PPI History } & $\begin{array}{c}\text { Number of Subjects } \\
\text { (\% Total Sample) }\end{array}$ & $\begin{array}{c}\text { Successful } \\
\text { Step-Down } \\
(\%)\end{array}$ \\
\hline Previous step-up & $70(59.8)$ & $54(77.1)$ \\
No previous single-dose PPI & $28(23.9)$ & $23(82.1)$ \\
Previous trial of step-down & $19(16.2)$ & $16(84.2)$ \\
\hline
\end{tabular}

from greater than single-dose to single-dose PPI therapy. Despite previous failure in these subjects (recurrence of symptoms requiring PPI dose escalation), 16 (84\%) successfully stepped-down to single-dose PPI and remained free of heartburn or acid regurgitation for the duration of the study.

Overall quality of life did not significantly change between baseline and 6-month follow-up. Mean ( \pm SD) SF-12 physical component summary scores were $31.54( \pm 9.88)$ at baseline and 29.9 ( \pm 8.31) at follow-up. SF-12 mental component summary scores were $45.3( \pm 11.9)$ at baseline and 40.8 ( \pm 10.6$)$ at follow-up. One question contained in the disease-specific quality of life measure, "During the past $4 \mathrm{wk}$, how severe has your pain been because of your gastrointestinal symptoms?" resulted in a significant increase between baseline and follow-up scores $(p<0.05)$. Disease severity did not significantly change in any other areas, including heartburn and acid regurgitation.

Univariate analysis of baseline demographic data and clinical information revealed that only the duration of PPI use before study enrollment predicted outcome. For every additional year of prior PPI use, subjects were 34\% more likely to fail the attempt to step-down to single-dose PPI therapy (Table 3).

Initial medication cost for this cohort was $\$ 115,570$ per year. Postintervention medication cost for this cohort was $\$ 54,520$ per year. The estimated direct cost for operation of the Heartburn Clinic was $\$ 27,342$ per year (salary and benefits for weighted effort of clinical pharmacist). Estimated cost-savings to the medical center was $\$ 33,708$ per year for the cohort examined.

Table 3. Predictors of Successful Step-Down

\begin{tabular}{|c|c|c|c|}
\hline & OR & SE & $p$ \\
\hline Age (yr) & 1.00 & 0.02 & 0.97 \\
\hline $\begin{array}{l}\text { Tobacco use (smokers compared } \\
\text { with nonsmokers) }\end{array}$ & 1.52 & 0.50 & 0.40 \\
\hline $\begin{array}{l}\text { Alcohol use (any use compared } \\
\text { with no use) }\end{array}$ & 0.74 & 0.52 & 0.56 \\
\hline Heartburn & 1.14 & 0.53 & 0.80 \\
\hline Regurgitation & 1.47 & 0.48 & 0.43 \\
\hline Dyspepsia & 1.64 & 0.55 & 0.37 \\
\hline $\begin{array}{l}\text { Initial PPI (lansoprazole compared } \\
\text { with omeprazole) }\end{array}$ & 3.74 & 1.06 & 0.22 \\
\hline $\begin{array}{l}\text { Site (Ann Arbor compared with } \\
\text { Toledo) }\end{array}$ & 1.10 & 0.48 & 0.84 \\
\hline $\begin{array}{l}\text { Duration of PPI before study (per } \\
\text { year of use) }\end{array}$ & 0.66 & 0.18 & 0.03 \\
\hline
\end{tabular}




\section{DISCUSSION}

The purpose of this study was to determine whether patients with symptoms of heartburn or acid regurgitation rendered asymptomatic on multiple-dose PPI therapy could be stepped-down to single-dose therapy. The results illustrate that nearly $80 \%$ of participants could be successfully managed with lower doses of PPIs. The success of step-down was predicted only by the duration of PPI use before the study, whereby longer duration was associated with greater recurrence of heartburn or acid regurgitation. All but one subject who failed step-down did so within the first 3 months of observation. Overall quality of life was not adversely impacted by this intervention, whereas sizeable cost savings were realized.

The majority of our study population was successfully stepped-down from greater than single-dose PPI therapy to single-dose PPI therapy. These results expand on our earlier study that showed that more than half of subjects in whom PPIs had alleviated reflux-type symptoms remained asymptomatic after discontinuation of PPIs and institution of either less-expensive or no medication (5). It is possible that the basis for both results is that less potent acid suppression is required for maintenance after symptom resolution has been achieved. Additionally, step-down was successful regardless of PPI history. Subjects who had been stepped-up from single-dose to greater than single-dose PPI for persistent heartburn or acid regurgitation were successfully steppeddown in a proportion similar to those who had not previously attempted single-dose PPI therapy. More surprisingly, subjects who had failed a previous trial of single-dose therapy were just as likely to successfully step-down as other subgroups. The only identifiable difference between the study protocol and previous attempts of step-down was the addition of the Heartburn Clinic, where the study pharmacist educated patients about GERD therapy, including lifestyle modifications and correct use of medication (including administration of PPIs $1 / 2 \mathrm{~h}$ before a meal).

Prior research has shown that patients with endoscopically documented erosive esophagitis require maintenance PPIs to prevent relapse $(15,16)$. These studies, however, do not represent the more universal population of patients with heartburn or acid regurgitation, who might not have esophagitis, and therefore should not be used to predict the most effective long-term management therapy of GERD in general. One of the novel aspects of our study is that we did not limit our study to subjects with erosive esophagitis, and therefore our results might be applicable to a more broad population of patients with reflux-type symptoms. It has also been shown that age is a significant factor of successful step-down therapy (5). It might be that older patients' perception of GERD symptoms is less severe compared with younger patients (17). In this study, no age significance was found; however, almost all of the subjects in this study experienced successful step-down therapy, resulting in a nonheterogeneous distribution of the final outcome. Peduzzi et al. have shown that it is essential to have at least 10 outcome events per variable evaluated in a logistic regression to avoid errors in the regression coefficients (12). Thus, the paucity of outcome events in this study might explain why no predictors for successful step-down could be identified $(13,14)$.

Limitations to this study include the paucity of young subjects and women, which decreases the generalizability of our results to non-veteran populations. Additionally, our patient population consisted of "real-world" subjects, who had been managed empirically with PPI therapy based solely on relief of symptoms of heartburn and acid regurgitation and who were inferred to have GERD without other objective measures, such as endoscopy or ambulatory $\mathrm{pH}$ monitoring. It is possible that some subjects might not have had GERD but some form of functional dyspepsia that responded to acid suppression therapy (7-10). This might have resulted in the finding that pain unrelated to heartburn or acid regurgitation increased in the study cohort with reduction of PPI dose. The rationale for greater than singledose PPI therapy was not recorded in most cases, thus it cannot be stated with certainty that all subjects required high-dose PPIs to alleviate their symptoms. Last, the cost analysis reported does not include indirect costs, such as office space, ancillary staff, administrative support, or direct costs that would be incurred in caring for symptomatic subjects. These omissions might also limit the applicability of this analysis to other healthcare settings.

In practice, it might be difficult to definitively confirm the diagnosis of GERD. Thus, we often empirically use acid suppression in patients with symptoms of heartburn and acid regurgitation because this is effective. However, this therapy might improve symptoms not only for GERD but also for functional dyspepsia and other disorders. As a result, errors in diagnosis might be made, thus labeling some patients incorrectly and encouraging the use of long-term acid suppression. This study points out that PPI dose might be titrated after initial symptom resolution.

In conclusion, this study revealed that the majority of patients rendered asymptomatic on greater than single-dose PPI therapy for symptoms of heartburn or acid regurgitation could be successfully stepped-down to single-dose therapy. The success of step-down was not limited to subgroups of our study population, but was instead broadly applicable. It is likely that PPI step-down management of patients with reflux-type symptoms will decrease management costs without adversely affecting quality of life.

\section{ACKNOWLEDGMENTS}

Supported by the U.S. Department of Veterans Affairs, Veterans Health Administration, Health Services Research and Development Service IIR 99-238-2, and in part by a grant from TAP Pharmaceuticals. JMI is also supported by a Faculty Development Award from the American College of Gastroenterology. 
Reprint requests and correspondence: John M. Inadomi, M.D., VA Ann Arbor Healthcare Systems, 111-D, 2215 Fuller Road, Ann Arbor, MI 48105.

Received Sep. 12, 2002; accepted May 12, 2003.

\section{REFERENCES}

1. Pharmacy Benefits Management Strategic Health Group. Contract adherence reports national reports: Q4FY99. Washington, DC: Department of Veterans Affairs, Veterans Health Administration, 2000.

2. Sontag S. Gastroesophageal reflux disease. Aliment Pharmacol Ther 1993;7:293-312.

3. Bell N, Hunt R. Role of gastric acid suppression in the treatment of gastro-oesophageal reflux disease. Gut 1992;33:118-24.

4. Wesdorp I, Dekker W, Festen HP. Efficacy of famotidine 20 $\mathrm{mg}$ twice a day versus $40 \mathrm{mg}$ twice a day in the treatment of erosive or ulcerative reflux esophagitis. Dig Dis Sci 1993;38: 2287-93.

5. Inadomi JM, Jamal R, Murata GH, et al. Step-down management of gastroesophageal reflux disease. Gastroenterology 2001;121:1095-100.

6. Nyren O, Adami H, Bates S, et al. Absence of therapeutic benefit from antacids or cimetidine in non-ulcer dyspepsia. N Engl J Med 1986;314:339-43.

7. Dobrilla G, Comberlato M, Steele A, Vallaperta P. Drug treatment of functional dyspepsia. A meta-analysis of randomized controlled clinical trials. J Clin Gastroenterol 1989;11: 169-77.

8. Talley NJ, Meineche-Schmidt V, Pare P, et al. Efficacy of omeprazole in functional dyspepsia: Double-blind, randomized, placebo-controlled trials (the Bond and Opera studies). Aliment Pharmacol Ther 1998;12:1055-65.
9. Farup PG, Wetterhus S, Osnes M, Ulshagen K. Ranitidine effectively relieves symptoms in a subset of patients with functional dyspepsia. Scand J Gastroenterol 1997;32:755-9.

10. Talley NJ, McNeil D, Hayden A, Piper DW. Randomized, double-blind, placebo-controlled crossover trial of cimetidine and pirenzepine in nonulcer dyspepsia. Gastroenterology 1986;91:149-56.

11. Colwell HH, Mathias SD, Pasta DJ, et al. Development of a health-related quality of life questionnaire for individuals with gastroesophageal reflux disease. A validation study. Dig Dis Sci 1999;44:1376-83.

12. Peduzzi P, Concato J, Kemper E, et al. A simulation study of the number of events per variable in logistic regression analysis. J Clin Epidemiol 1996;49:1373-9.

13. Peduzzi P, Concato J, Feinstein AR, Holford TR. Importance of events per independent variable in proportional hazards regression analysis. II. Accuracy and precision of regression estimates. J Clin Epidemiol 1995;48:1503-10.

14. Concato J, Peduzzi P, Holford TR, Feinstein AR. Importance of events per independent variable in proportional hazards analysis. I. Background, goals, and general strategy. J Clin Epidemiol 1995;48:1495-501.

15. Hallerback B, Unge P, Carling L, et al. Omeprazole or ranitidine in long-term treatment of reflux esophagitis. The Scandinavian Clinics for United Research Group. Gastroenterology 1994;107:1305-11.

16. Vigneri S, Termini R, Leandro G, et al. A comparison of five maintenance therapies for reflux esophagitis. N Engl J Med 1995;333:1106-10.

17. Fass R, Pulliam G, Johnson C, et al. Symptom severity and oesophageal chemosensitivity to acid in older and young patients with gastro-oesophageal reflux. Age Ageing 2000;29: 125-30. 